IJADR

International Journal of Alcohol and Drug Research

The Official Journal of the Kettil Bruun Society for Social and Epidemiological Research on Alcohol

doi: 10.7895/ijadr.v4i1.189

IJADR, 2015, 4(1), 21 - 25

ISSN: 1925-7066

\title{
Addiction-as-a-kind hypothesis
}

\author{
Petri Ylikoski ${ }^{1}$ and Samuli Pöyhönen ${ }^{2}$ \\ ${ }^{1}$ Department of Social Research/Sociology, University of Helsinki, Finland \\ ${ }^{2}$ Social and Moral Philosophy, University of Helsinki, Finland
}

\begin{abstract}
The psychiatric category of addiction has recently been broadened to include new behaviors. This has prompted critical discussion about the value of a concept that covers so many different substances and activities. Many of the debates surrounding the notion of addiction stem from different views concerning what kind of a thing addiction fundamentally is. In this essay, we put forward an account that conceptualizes different addictions as sharing a cluster of relevant properties (the syndrome) that is supported by a matrix of causal mechanisms. According to this "addiction-as-a-kind" hypothesis, several different kinds of substance and behavioral addictions can be thought of as instantiations of the same thing-addiction. We show how a clearly articulated account of addiction can facilitate empirical research and the theoretical integration of different perspectives on addiction. The causal matrix approach provides a promising alternative to existing accounts of the nature of psychiatric disorders, the traditional disease model, and its competitors. It is a positive addition to discussions about diagnostic criteria, and sheds light on how psychiatric classification may be integrated with research done in other scientific fields. We argue that it also provides a plausible approach to understanding comorbidity.
\end{abstract}

In recent years, the concept of addiction, as it is used both in research and in policy contexts, has been expanded to include new behaviors (e.g., gambling, eating, sex, shopping, exercise, even internet use). There has also been critical discussion about the value of a concept that covers so many different substances and activities. In this essay, we present an account of the conditions under which several kinds of substance and behavioral addictions can be thought of as instantiations of the same thing-addiction. We call this account the "addiction-as-a-kind" hypothesis. The account describes what addiction would look like if it were a legitimate psychiatric kind. We call it a hypothesis because it is an empirical question whether different addictions really constitute such a kind. In this essay, we will not assess the empirical support for the hypothesis, but will focus on describing some of its general characteristics. In other words, we describe what a general theory of addiction could look like.

The paper is structured as follows. First we frame our theory by briefly considering some difficulties with the traditional disease model and its alternatives. Then we present our addiction-as-a-kind hypothesis and the underlying causal matrix view of psychiatric kinds. The final section discusses the issue of comorbidity and, based on the hypothesis, offers some suggestions for addiction research.

\section{The Problem With the Traditional Disease Model}

There has been a longstanding debate in psychiatry concerning the nature of psychiatric disorders, with still no agreement on what psychiatric illnesses actually are. The traditional disease model that conceptualizes psychiatric illnesses based on a model drawn from medicine is still commonly accepted (Kincaid \& Sullivan, 2010; cf. Leshner, 1997). This model builds on essentialist thinking, as it presumes that psychiatric illnesses can be understood as well-determined entities having a few causal core properties, which in turn explain the rest of their properties. Moreover, it is usually assumed that this essence must lie within the afflicted individual. One example of a medical condition satisfying the essentialist model is Huntington's disease. The genetic basis of Huntington's forms its causal core, in the sense that it can be used to explain many of the properties of the condition.

Psychiatric disorders create difficult problems for the medical model. Most psychiatric disorders seem to have multiple contributing and sustaining causes (Murphy, 2010). Furthermore, in many cases there is a continuum of severity of the disorder, without a clear-cut established difference between the disorder and the normal state. Both considerations are problematic for an account that aims to identify the disease with a single well-defined etiological cause that is clearly separate from normal functioning.

Correspondence: Samuli Pöyhönen, Social and Moral Philosophy, P.O. Box 24, 00014 University of Helsinki, Finland. Telephone: +358-50-4154926; Fax: +358-9191 28333, E-mail: samuli.poyhonen@helsinki.fi

Financial support: This research received financial support from the University of Helsinki and the Academy of Finland

Keywords: addiction, natural kind, mechanism 
Furthermore, the traditional medical model seems to be implicitly biased in favor of "biological" causes, thus making it difficult to integrate an understanding of the influence of psychological and social mechanisms with knowledge of neural and genetic processes (Kincaid \& Sullivan, 2010).

These problems suggest that the traditional disease model is not a good starting point for developing a general theory of addiction. However, no consensus exists on an alternative approach. Psychiatric classification systems such as DSM and ICD are purely descriptive, and while serving several administrative and clinical purposes, they are a poor foundation for a scientific study of the causes of psychiatric disorders (Adam, 2013; Craddock \& Owen, 2010; Murphy, 2006). Likewise, we do not think that accounts that view addiction as normal choice-behavior under exceptional circumstances (Heyman, 2009) can really solve the problem, as they have trouble incorporating findings about the cognitive and neural processes underlying addictive behavior.

In debates about addiction (and other mental disorders), the traditional disease model often serves as a straw man that is easy to criticize. Because a positive alternative to it is missing, the failure of the traditional disease model fuels skepticism about the whole notion of addiction. The purpose of our addiction-as-a-kind hypothesis is to provide a viable alternative to the traditional disease model of addiction. A clearly articulated account of addiction as a psychiatric kind may aid in facilitating discussions about the diagnostic criteria, definition, and classification of addiction, insofar as it may provide a context for developing substantive theories about the disorder. It would also conceptually facilitate the integration of neuroscientific, psychological, and social scientific insights into a substantive synthetic theory of addiction. It is apparent that many of these benefits do not depend on the addiction-as-a-kind hypothesis being true. A false theory can also be fruitful in directing research (see Wimsatt, 2007), whereas a complete lack of a theory rarely is.

\section{The Addiction-as-a-Kind Hypothesis}

Our replacement for the traditional model is based on the mechanistic property cluster view of kinds (Boyd, 1999), which has been developed in the philosophy of science over the last 30 years, and has more recently been applied to psychiatric kinds (Kendler, Zachar, \& Craver, 2010; Kuorikoski \& Pöyhönen, 2012; Murphy, 2006). We believe that this account provides a reasonable middle way between the medical model, the pragmatic kinds theory (Zachar, 2003), and more minimalist approaches that conceive of having a mental illness as nothing more than meeting diagnostic criteria.

In our proposal, a psychiatric kind consists of two elements:

(i) a cluster of typical properties (etiology, symptoms, response patterns to treatments, etc.) that identify the disorder (we call this the syndrome), and (ii) a matrix of causal mechanisms that are responsible for the co-occurrence of the properties in the cluster.

In this account, a psychiatric disorder is identified on the basis of a cluster of co-occurring properties, rather than on a single genetic, physical, or psychological cause that produces the cluster of properties typical of the disorder. Thus, the disorder is identified by behavioral criteria, but it is solidly rooted in causal mechanisms that are responsible for the co-occurrence of the typical properties.

This approach saves the basic motivation of the traditional disease model, while discarding its restrictive essentialist assumption. The classification of disorders is still anchored in underlying causal mechanisms that provide a basis both for the explanation of the symptoms and for designing effective therapeutic interventions. Hence, the advantages of the traditional model are not lost. The new account can also incorporate the traditional disease model as a special case where there is one specific underlying cause of the disease; it is therefore possible that the traditional model might apply to some (but probably only a few) psychiatric disorders.

However, in contrast to the traditional model, the new nonessentialist account allows for some heterogeneity both in (a) the causal pathways producing the cluster of symptoms typical of the disorder (cf. Blaszczynski \& Nower, 2002) (i.e., different combinations of the matrix can produce the same disorder profile), and (b) the property cluster itself (as the different combinations of contributing mechanisms would presumably produce slightly different outcomes). Moreover, not all causes of addiction must lie within the afflicted individual (see below, 'Comorbidity and Other Sources of Heuristic Value'). It is also notable that in this account, the psychiatric kind need not be timeless. This accommodates the possibility that the symptoms of a disorder may change over time or be slightly different in different populations (Hacking, 2007). To be recognized as the same disorder, it need only have a cluster of symptoms that remains robust and a set of underlying mechanisms that are sufficiently similar. In the rest of this section, we show how these general ideas can be applied to the case of addiction.

Tentatively, addiction can be characterized as a selfadministered activity that is both harmful and difficult to quit or control. The idea of this general formulation is that it covers both substance and behavioral addictionssubstance use can also be conceived of as an activity-thus making it possible to formulate the addiction-as-kindhypothesis as generally as possible. The hypothesis states that addiction is a general kind that covers various forms of addiction that are currently often defined by their object (i.e., the substance or activity).

The idea is that all forms of addiction share the same basic characteristics: the relevant cluster of symptoms-the syndrome (see the end of this section) — is sufficiently similar, because the clustering of these properties is explained by the functioning of a set of mechanisms 
belonging to the causal matrix of the disorder. This shared cluster of symptoms between addictions is supposed to distinguish them from other similar conditions, such as obsessive-compulsive disorder (see discussion of Goodman, 2008, below). Naturally, specific addictions also have certain properties specific to the particular form of addiction. These characteristics are consequences of the nature of particular addictive substances or behaviors. However, while specific addictions - and individual cases of addiction-might not share all properties of the syndrome, it is expected that the underlying mechanisms would provide a non-ad-hoc explanation of these absences.

Unlike the medical model, our causal matrix approach does not require that addiction be defined on the basis of a single mechanistic factor (e.g., DA system), and is compatible with models that see addiction as being maintained by a complex collection of neural, psychological, and social factors (e.g., Goodman, 2008; Orford, 2001; Shaffer et al., 2005; West, 2006). It also makes it plausible that the subkinds of addiction can have subtypes with their own profiles (e.g., the pathway model of Blaszczynski \& Nower, 2002).

Our causal matrix approach suggests a way to assess the plausibility of the addiction-as-a-kind hypothesis: the idea of addiction as a general kind makes sense to the degree that a sufficient similarity between different forms of addiction can be found (in contrast to other classes of disorders, for example OCD). There should be similarity both in the cluster of properties that characterize the syndrome and the mechanisms responsible for it. Consequently, support for the hypothesis is a matter of degree: the greater the similarities among the various forms of addiction, the better the hypothesis is supported. In this sense, the idea of addiction as a general kind is an empirical hypothesis to be tested. However, in contrast to the traditional disease model, in our theory the fate of the kind "addiction" does not depend on the existence of a set of necessary and sufficient conditions that capture the (actually existing) essence of the disorder. Instead, our mechanistic cluster theory suggests that the value of the general concept of addiction is to be judged by its usefulness in theory development, as well as its advice for developing therapies for different sorts of addictions. In the last section of this essay, we discuss this heuristic usefulness of the general concept of addiction in more detail.

While the extent of our expertise does not allow for the formulation of a substantial empirical theory of addiction, we think Aviel Goodman (2008) provides a promising sketch for such a theory, which can be used to illustrate our idea. Goodman does not define addiction by referring to the properties of certain addictive substances or a malfunctioning brain structure. According to him, different addictions are characterized by similar features, such as the typical course of the illness, the experience of tolerance, the withdrawal phenomena, the tendency to relapse, and the specific patterns of comorbidity. Goodman's list is a rich description of the syndrome associated with addiction, and we think that if it is possible to arrive at an empirical characterization of an addiction syndrome that resembles Goodman's characterization, we have a good basis for treating addiction as a legitimate kind.

\section{How Mechanisms Make the Difference}

According to our proposal, the core of addiction research is in the study of the matrix of causal mechanisms that (i) make individuals vulnerable to addiction, (ii) are involved in the development of addiction, and (iii) sustain the addiction and make it difficult to quit. The inclusion criteria for the matrix are quite liberal, and may cover a large group of causal factors that are significant differencemakers in terms of the development and characteristics of the addiction syndrome. Unlike mere lists of variables associated with addiction, causal-mechanistic knowledge gives us an understanding of how causally relevant factors produce their effects (Craver, 2007; Hedström \& Ylikoski, 2010). Such understanding provides a solid basis for both a proper understanding of addiction and the development of effective therapeutic interventions. Therefore, research should go beyond identifying correlations and characteristic properties of phenomena, and aim to reveal the nature of causal dependencies between variables.

A central aspect of the causal matrix approach is that the relevant mechanisms can work at various levels or scales (Ylikoski, 2012). Therefore, the approach does not privilege any specific scale of causal interaction in addiction research. What is crucial is whether the processes are relevant difference-makers to the condition, not what size they are. Our account can therefore incorporate sub-personal neural processes, cognitive mechanisms that are attributed to whole persons, and social processes that characterize the interaction of an individual with her social environment, without giving metaphysical or methodological privilege to any of them.

Another implication of the causal matrix view is that it makes sense of how understanding pathological mechanisms builds on knowledge of normal functioning: the same basic mechanisms are at work in both cases. In contrast to the traditional view, in which it is sufficient to identify the pathogen or malfunction to explain the disease, the causal matrix approach suggests that it is not enough to identify malfunctions in, for example, the dopamine system or impulse inhibition; one must also understand how these abnormalities lead to the development and stability of the syndrome. Hence, addiction research should pay close attention to the scientific study of the normal functioning of the systems in question. This would facilitate the integration of psychiatric classification with research on non-pathological processes conducted in other scientific fields. Such integration can make new knowledge available across the boundaries of research fields and contribute to the development of a comprehensive account of addiction, a theory that would integrate biological, psychological, and social mechanisms.

In addition, focusing on a matrix of causal mechanisms clarifies the continuity between the normal and the pathological. With this approach, there need not be a clearcut threshold for having or not having a disorder; people 
involved in an activity could be classified along a continuum (e.g., casual users, problem users, addicts). The cases can be evaluated according to two dimensions: (1) how harmful the activity is, and (2) how difficult it is to control or quit. The former provides a natural dimension for psychiatric importance, while the latter is a natural measure of the severity of the addiction. The judgment of what constitutes a threshold for psychiatric treatment is ultimately a value judgment (as are all medical judgments). Thus, the causal matrix view makes it possible to recognize important similarities between, for example, being in love (Reynard et al., 2010) and gambling addiction, without automatically making both psychiatric disorders.

Finally, while there are reasons to suspect that some people are more vulnerable to addiction than others (due to genetic (Bierut, 2011), developmental, and environmental factors), the causal matrix approach does not support the idea of an "addictive personality." As the mechanisms underlying the addiction are not external pathogens, but instead the (abnormal) workings of "normal" processes, one cannot assume that only some people are vulnerable (although they might be at higher risk).

\section{Comorbidity and Other Sources of Heuristic Value}

A recurring observation about addictions is the high comorbidity (Heyman, 2009; Petry et al., 2005). If a person has one form of addiction, there is an increased probability that he or she also has, has had, or will have some other form of addiction. From the mechanistic point of view, this is to be expected: as the matrix of underlying mechanisms is the same, comorbidities between different forms of addiction are not surprising. In fact, it makes sense to study why there is not more comorbidity between different forms of addiction.

Another form of comorbidity is with other mental disorders. For traditional accounts of psychiatric classification, unexplained comorbidities have been a nuisance (cf. Cramer, Waldorp, van der Maas, \& Borsboom, 2010), but within the causal matrix approach they are naturally accommodated. If the matrix of the underlying mechanisms of other psychiatric disorders (for example, depression) has common elements with that of addiction, some comorbidity is to be expected. Even if the matrices are largely disjoint, there can be some comorbidity, because other mental disorders may act as contributing factors, causally triggering some part of the matrix underlying the disorder of interest.

This has an important consequence for psychiatric classification. Rather than a nuisance, comorbidity should be seen as a fact and a heuristic cue. That is, comorbidity between two disorders may act a source of hypotheses regarding the shared or otherwise related structures and mechanisms underlying the disorders in question. Thus, psychiatric classification should not assume that mental disorders are exclusive of each other. As the underlying mechanisms are not disjoint, one should not expect that disorders are completely disjoint either.
Another major added value of the addiction-as-a-kind idea is the heuristic guidance it provides for addiction research: The key idea is that what is known about one form of addiction should suggest testable hypotheses concerning other forms of addiction. Thus, it makes sense to study different addictions together and conduct more comparative studies. This is the underlying rationale for tentatively expanding the notion of addiction to new activities. Sometimes the strategy is successful and sometimes not, but the point is that studying the underlying mechanisms provides a principled way to test the idea.

Another interesting heuristic possibility has been suggested by Alex Blaszczynski and Lia Nower (2002; Milosevic \& Ledgerwood, 2010). They propose that the development of gambling addiction occurs along three distinct causal pathways, corresponding to differences in individuals' physiological, cognitive, and affective properties and dissimilarities in their social settings. Moreover, these processes often involve interactions among factors at the different scales. The interactions can lead to the development of self-enforcing "traps" or "loops," where engaging in addictive behavior reduces the value of other behavioral options, or where the adverse social consequences of addictive behavior lead to secondary processes (e.g., psychological stress) that in turn enforce addictive behavior. We suggest that the different pathways underlying behaviorally similar addictions could be used to identify subforms of addiction, and that similar subforms could be found across several different substance and behavioral addictions. Identifying these subforms might allow for richer and more reliable inferences regarding particular addictions than does identifying variants of the disorder only by a particular substance or activity.

Similar heuristic ideas might help the development of therapeutic interventions. The mechanism-based approach suggests that it makes sense to focus on treatments that target specific mechanisms (Potenza, Sofuoglu, Carroll, \& Rounsaville, 2011), rather than on general solutions to "addiction." This idea also provides a rationale for the expectation that interventions targeting certain general mechanisms could work for a large variety of addictions.

Finally, the search for mechanisms constitutes a strategy for integrating different theoretical perspectives on addiction (Kovac, 2012; Orford, 2001; West, 2006). Neither neuroscientific theories of addiction nor choicebased approaches building on common-sense psychology should have a privileged position in describing addiction as a phenomenon (Kalant, 2010). While neural processes are always involved in addiction, it should not be assumed a priori that pharmacological interventions should be somehow privileged. Similarly, while it is legitimate to apply folk-psychological agency concepts (like choice or desire), a full understanding of the phenomenon of addiction requires going beyond these by connecting the choice-theoretical perspective (Heyman, 2009) to knowledge about neural systems, the relevant cognitive biases, and social dynamics (Redish, Jensen, \& Johnson, 2008; Volkow, Baler, \& Godstein, 2011). By giving up the strong dichotomy between neuroscientific approaches and the agency perspective, the mechanism-based approach 
provides a way to work towards a more psychologically realistic picture of human agency.

\section{References}

Adam, D. (2013). Mental health: On the spectrum. Nature News, 496(7446), 416.

Bierut, L. J. (2011). Genetic vulnerability and susceptibility to substance dependence. Neuron, 69(4), 618-627.

Blaszczynski, A., \& Nower, L. (2002). A pathways model of problem and pathological gambling. Addiction, 97(5), 487-499.

Boyd, R. (1999). Homeostasis, species, and higher taxa. In R. A. Wilson (Ed.), Species: New interdisciplinary essays (pp. 141-185). Cambridge, MA: MIT Press.

Borsboom, D., \& Cramer, A. (2013). Network analysis: An integrative approach to the structure of psychopathology. Annual Review of Clinical Psychology, 9, 91-121.

Craddock, N., \& Owen, M. J. (2010). The Kraepelinian dichotomy-going, going . . . but still not gone. The British Journal of Psychiatry, 196, 92-95.

Cramer, A., Waldorp, L., van der Maas, H., \& Borsboom, D. (2010). Comorbidity: A network perspective. Behavioral and Brain Sciences, 33, 137-193.

Craver, C. (2007). Explaining the brain: Mechanisms and the mosaic unity of neuroscience. Oxford, U.K.: Oxford University Press.

Goodman, A. (2008). Neurobiology of addiction: An integrative review. Biochemical Pharmacology, 75(1), 266-322.

Hacking, I. (2007). Kinds of people: Moving targets. Proceedings of the British Academy, 151, 285-318.

Hedström, P., \& Ylikoski, P. (2010). Causal mechanisms in the social sciences. Annual Review of Sociology, 36, 49-67.

Heyman, G. M. (2009). Addiction: A disorder of choice. Cambridge, MA, United States: Harvard University Press.

Kalant, H. (2010). What neurobiology cannot tell us about addiction. Addiction, 105(5), 780-789.

Kendler, K. S., Zachar, P., \& Craver, C. (2010). What kinds of things are psychiatric disorders? Psychological Medicine, 41(06), 1143-1150.

Kincaid, H., \& Sullivan, J. (2010). Medical models of addiction. In D. Ross, H. Kincaid, D. Spurrett, \& P. Collins (Eds.), What is addiction? (pp. 353-376). Cambridge, MA, United States: MIT Press.

Kovac, V. B. (2013). The more the "Merrier": A multisourced model of addiction. Addiction Research \& Theory, 21(1), 19-32.

Kuorikoski, J., \& Pöyhönen S. (2012). Looping kinds and social mechanisms. Sociological Theory, 30(3), 187205.

Leshner, D. (1997). Addiction is a brain disease, and it matters. Science, 278, 45-47.

Milosevic, A., \& Ledgerwood, D. M. (2010). The subtyping of pathological gambling: A comprehensive review. Clinical Psychology Review, 30(8), 988-998.

Murphy, D. (2006). Psychiatry in the scientific image. Cambridge, MA, United States: MIT Press.
Murphy, D. (2010). Explanation in psychiatry. Philosophy Compass, 5(7), 602-610.

Orford, J. (2001). Addiction as excessive appetite. Addiction, 96(1), 15-31.

Petry, N. M., Stinson, F. S., \& Grant, B. F. (2005). Comorbidity of DSM-IV pathological gambling and other psychiatric disorders: Results from the National Epidemiologic Survey on Alcohol and Related Conditions. Journal of Clinical Psychiatry, 66(5), 564-574.

Potenza, M. N., Sofuoglu, M., Carroll, K. M., \& Rounsaville, B. J. (2011). Neuroscience of behavioral and pharmacological treatments for addictions. Neuron, 69(4), 695-712.

Redish, A. D., Jensen, S., \& Johnson, A. (2008). A unified framework for addiction: Vulnerabilities in the decision process. Behavioral and Brain Sciences, 31(4), 415-437.

Reynaud, M., Karila, L., Blecha, L., \& Benyamina, A. (2010). Is love passion an addictive disorder? The American Journal of Drug and Alcohol Abuse, 36(5), 261-267.

Shaffer, H. J., LaPlante, D. A., LaBrie, R. A., Kidman, R. C., Donato, A. N., \& Stanton, M. V. (2004). Toward a syndrome model of addiction: Multiple expressions, common etiology. Harvard Review of Psychiatry, 12(6), 367-374.

Volkow, N. D., Baler, R. D., \& Goldstein, R. Z. (2011). Addiction: Pulling at the neural threads of social behaviors. Neuron, 69(4), 599-602.

West, R. W. (2006). Theory of addiction. Hoboken, NJ, United States: Blackwell Publishing.

Wimsatt, W. (2007). Re-engineering philosophy for limited beings: Piecewise approximations to reality. Cambridge, MA, United States: Harvard University Press.

Ylikoski, P. (2012). Micro, macro, and mechanisms. In H. Kincaid (ed.), The Oxford handbook of philosophy of the social sciences (pp. 21-45). Oxford, U.K.: Oxford University Press.

Zachar, P. (2003). The practical kinds model as a pragmatist theory of classification. Philosophy, Psychology and Psychiatry, 9, 219-227. 